\title{
Milieu Therapy under the Primary \\ Caretaker System at the University of Michigan's Children's Psychiatric Hospital
}

\author{
Wayne R. Kutschinski \\ University of Michigan's Children's Psychiatric Hospital
}

\begin{abstract}
The inpatient service at the University of Michigan's Children's Psychiatric Hospital is a multidisciplined program whose purpose is to create a therapeutic milieu in which emotionally disturbed children can live while being effectively treated. The work of the primary staff as milieu therapist on the ward is a key factor in the success or failure of many cases. This paper describes the therapeutic milieu, the primary caretaker system of staffing, and the specific role of the primary staff as milieu therapist at the hospital.
\end{abstract}

The intent of this paper is twofold. First, I wish to give an overview of the inpatient program at Children's Psychiatric Hospital of the University of Michigan. Included will be outlines of some of the institution's structural systems: the building, the administration, the multiple theories subscribed to, the team approach used, and the therapeutic milieu created. The primary focus will then shift to the role, in relation to these institutional systems, played by the psychiatric care worker (P.C.W.) as a primary staff and milieu therapist in the treatment of the children who are inpatients at the hospital.

Second, this paper is intended as a foundation upon which to expand in later writings the methods I have found most effective in performing successful milieu therapy. A case study will be used to illustrate these methods and to introduce my theory that an eclectic approach synthesizing what is useful from many different theories of psychology is the most sensible and successful approach that can be made to discovering and improving methods for treating patients who are emotionally disturbed.

Mr. Kutschinski is Psychiatric Care Worker II, University of Michigan's Children's Psychiatric Hospital. Please send all correspondence to Wayne Kutschinski, 1411 Traver, Ann Arbor, Michigan 48105. 


\section{The Therapeutic Milieu}

The therapeutic milieu at Children's Psychiatric Hospital (C.P.H.) can be described as a collection of the various areas of the hospital inpatient environment to which patients are exposed and by which exposure the patients receive various forms of therapeutic help for their problems (which by definition are psychiatric).

These areas include the ward (the living unit, which constitutes the patient's surrogate home while an inpatient at C.P.H. and contains 12 bedrooms, a dining room with attached kitchenette and snack room, bathrooms and shower rooms, large and small playrooms, a nursing station and a staff room); the doctors' offices, where psychotherapy is performed; the school (six classrooms); the activities therapy area, which is comprised of three occupational shops; the kitchen; and the recreational therapy department, complete with its own gymnasium, swimming pool, exercise room, playground, and softball field. All of these facilities are parts of the therapeutic milieu and are contained in the building and surrounding grounds of C.P.H.

Also included in this collection, by extension (through services offered by C.P.H.), are the patients' own families and those families' communities, as well as the community of Ann Arbor itself. The former by virtue of its exposure to the social worker assigned to work with a particular family and the latter by virtue of its structured exposure to the patient by his primary caretaker (P.C.).

Each part of the therapeutic milieu is rendered "therapeutic" by virtue of the person under whose jurisdiction the patient is placed for periods of time ranging from 2 to 3 hours per week (the psychotherapist), to 10 to 15 hours per week (teachers, occupation and recreational therapists), to 30 hours per week (the primary caretaker). Each one is responsible for making the time he spends with the patient therapeutic and has at his disposal a variety of tools and techniques. Naturally, the specific role performed by each member of the therapeutic milieu in relation to the patient varies according to the area he performs in. Hence, the therapist conducts psychotherapy; the occupational therapist, occupational therapy; the teacher, special education; and the social worker, family therapy. To the P.C.W. and the nurse falls the role of primary caretaking and milieu therapy. Each child, therefore, is treated by a team of adults, and it is this group that comprises the child's "therapeutic team." Each team meets once per week during the patient's stay at C.P.H. to discuss his behavior in each area and the meaning of his behavior (diagnosis), to plan and co- 
ordinate treatment strategies, to set long- and short-term goals, to plan for discharge, etc. The manner in which the patient's behavior is interpreted, diagnosed, and treated varies from case to case and team to team depending on such individual factors as (a) the perceptual skills of the team members, (b) the communication skills of the team members, (c) the team members' interpretive and diagnostic skills, and (d) the theoretical and diagnostic positions of the team members, plus a host of other personality factors that come into play during group decision-making processes.

There are three distinctly institutionalized aspects of treatment of children at C.P.H. that ought to be highlighted. Each of these aspects has a great influence on the determination of the manner in which milieu treatment and the team concept are administered and, therefore, on the success or failure of the treatment of each child.

The first aspect involves the clearly delineated psychoanalytic orientation of the institution in terms of theory and ideology. What mental health is and what emotional disturbance is are defined by the theory. The focus of treatment is through the psychoanalytic lens, which determines to a large degree what is considered a therapeutic and/or countertherapeutic experience for the patient. In a like manner is progress measured and recorded. Case discussions and writings not surprisingly are usually couched in the language of psychoanalytic theory as well. Finally, it is worth noting that psychoanalytic theory is designed primarily for application to individuals and perhaps their families. Seldom does it attempt to deal with more global political, sociological, or economic issues independent of the individuals themselves. It is against the psychoanalytic model that all discussions of diagnosis, treatment techniques, management plans, and goals and disposition of the case are measured at C.P.H.

A second aspect of milieu treatment involving an institutionalized format has been the introduction and implementation of the Developmental Profile as the chief data-collecting and -digesting diagnostic tool at C.P.H. The Developmental Profile was conceived by Anna Freud [1] and developed and refined first at The Hampstead ChildTherapy Clinic in London, England. Dr. Humberto Nagera, the inpatient director at C.P.H., was heavily involved in the program's evolution at the clinic in London and introduced the program at C.P.H. The program is psychoanalytically oriented. It is an outline of the patient's development and pathology and contains the following major categories of scrutiny before and during the patient's admission and residence at C.P.H.: 
I. Reasons for referral

II. Description of the child

III. Family background and personal history of the child

IV. Significant environmental circumstances

V. Assessment of development

A. Drive development

B. Libido distribution

C. Cathexis of objects

D. Aggression

E. Ego and superego development

F. Ego defenses

G. Affect

H. Superego

I. Development of the personality

VI. Assessment of fixation and regression points

VII. Dynamic and structural assessment of major conflicts

VIII. General characteristics

IX. Diagnosis [2]

A Developmental Profile is written for each patient within 2 to 4 months after admission and approximately every 5 to 8 months thereafter for the duration of his stay. Each profile is presented in an hourlong discussion with team members and other members of the milieu, as well as the case supervisor and at least one member of the senior staff at C.P.H. Needless to say, within the confines of psychoanalytic theory, the Developmental Profile is a very thorough and comprehensive document and can be used extensively by members of the team in their work with the child.

A third institutional determinant of milieu treatment and team management involves the fact that C.P.H. is a hospital and part of a large university medical center, and the so-called "medical center model" of administration prevails. The consequences of this to the team and to the milieu are at least twofold: (1) The priorities are, in descending order, (a) training of university medical doctors and doctoral students as psychiatrists and clinical psychologists, (b) research into emotional disturbances and their "causes and cures," and (c) the treatment of the patient. (2) In terms of hierarchy (decision making, authority, status, income, etc.), medical doctors in general hold the highest positions, with clinical psychologists in the "titled aristocracy," while social workers, teachers, occupational therapists, recreational therapists, nurses, and psychiatric care workers descend below them in the approximate order listed. The combination of these two factors have some interesting results, which will be mentioned in the discussion of the P.C.W. as milieu therapist and later in the conclusion of the paper. 


\section{The Primary Caretaker System}

The primary caretaker system of child care and management was conceived by Dr. Humberto Nagera and is currently administered by Dr. Michael Colman at C.P H. [3] . Its purpose is to provide each child in the inpatient service (ages 5-13) with his own psychiatric care worker or nurse, whose functions would include filling the role of "surrogate parent" during the often long separation of the child from his real parents during treatment. Each primary caretaker is assigned to work with two children on a permanent basis. Thus the working prototype results in a single-parent (surrogate) family of three members. This model has been supplemented on the fourth-level inpatient unit at C.P.H. by linking two such families together to form a twoparent, four-child extended family. Sometimes the resulting grouping contains two men or two women, but more often it has one man and one woman. In addition, a third P.C.W. or nurse (i.e., a ward staff) is added to this extended family of six as a back-up staff with the function of working with the children of one or the other of the primary staff when that person has a day off. This third person corresponds roughly to a favorite aunt or uncle, in the sense that he or she maintains a continuity of parenting for the child that is acceptable and consistent with that of the parent surrogates in their absence.

Obviously, a primary caretaker cannot and is not expected to replace the child's own parents forever. And most parents are encouraged to visit their children regularly and to take them home on the weekend in order to preserve the original bond between them. This arrangement allows time for the development of an appreciation for, and an adjustment to, changes in the family that inevitably occur during the course of treatment while the child is absent from the home. In fact, it is crucial for this original parent-child bond to remain if the child is to be successfully integrated back into his family upon discharge. In some cases, however, this bond is not maintained, and the child is not returned home or the reintegration proves disastrous.

\section{The Role of the Parent Surrogate}

A primary caretaker, or "my primary staff" as they are called by their assigned children, fulfills many important and complex functions simultaneously both within and without the milieu.

A primary caretaker is required first of all to form an intimate therapeutic relationship with his patient so that he can adequately carry out his other surrogate functions. The patient must come to 
highly value and appreciate, in a positive sense, his primary staff. An attitude such as this provides motivation for change in that the patient will want to please his staff and maintain his good will and favor and will experience anxiety and/or guilt when he incurs, by his misbehavior, the displeasure of his staff. Hence, the staff serves as a consolidator of appropriate behavior and an agent of change (of inappropriate behavior) by skillful use of himself, of his favor, and of his attention as a selective reinforcer and shaper of new behavior. The staff also provides constant reliable feedback to the child regarding his progress while in treatment through discussion (life space interviews) and consistently applied and enforced behavioral limit setting appropriate for his age, stage of development, personal needs, etc. [4].

Second, the primary staff must act as an appropriate role model with whom the patient feels free to identify and whose behavior the patient can emulate, especially where previous role modeling of such adult behavior has been lacking in the child's life. If a child is to grow and change along healthy developmental lines, he needs to be offered forms of behavior alternative to those that have stimulated or contributed to whatever arrests in development and conflicts he is experiencing. He needs to know what is expected of him, what is acceptable behavior, and in what areas he needs to change. It is also very important that someone be available to give him feedback and demonstrate these behaviors while he endeavors to approximate them. That person is his staff. Since much internalized behavior is a result of assimilation and accommodation of schemes of action in the Piagetian sense, the importance of good role modeling can be easily seen. The child can learn to behave properly only when he understands how to, and he can understand only that which he has experienced first in his milieu [5].

The primary staff must also attend to the needs of his patients in their activities of daily living. This includes all of the day-to-day tasks every parent performs in the course of maintaining his or her children's physical well-being. He must be sure that the child is properly clothed and fed, that he bathes, combs his hair, and brushes his teeth; that he attends all of his scheduled activities; and that he receives proper amounts of exercise and rest. All of these nurturing activities during the final 5 or 6 working hours of the child's day are the primary caretaker's responsibility. They convey a message of interest and caring for the child and provide regular times for special interaction between the child and his staff. They also provide many areas in which the child's disturbances and conflicts may be acted out and 
dealt with on a day-to-day continuous basis and thus both present opportunity for milieu therapy to be performed and give information and clues to the staff concerning the progress of the child, his current mental state, and fixation in his pathology.

In addition to caring for the patient's needs for physical nurturing, the staff must also act as a social chairperson. In this role, the staff plans and schedules ward events and activities in the evening and on the weekends that will be therapeutic as well as fun for the child. $\mathrm{He}$ must be sensitive to the specific needs, skills, and preferences of his patient and facilitate the use of these activities in a manner that will provide the child with the corrective emotional experiences he needs. These activities can be designed to focus on any of a great number of potential problem areas: adult-child interactions, peer interactions, solitary activity, ego development, superego development, impulse control, self-esteem, cathexis of objects, etc. Again, opportunities for corrective emotional experiences must be emphasized when structuring activities. Further, these must be chosen in such a way as to allow the child to act out his disturbance so that therapeutic intervention may be made without undue stress or loss of self-esteem and without permanent diminution of the cathexis of the child to his staff.

Activities available include various arts and crafts (model building and ward decoration, for example), games of fine and large motor movement (e.g., softball, pool), quiet board games (e.g., Monopoly, Candyland), swimming, cooking, chores, television, books, and birthday parties.

Inherent in the surrogate parent's role as social chairperson is the additional responsibility of planning activities for his or her patient in the community outside of the hospital. This provides a wider variety of activities than are available within the hospital and similar therapeutic opportunities. Moreover, these outside activities are necessary to facilitate the child's eventual return to a home living situation, in which he will be expected to live in a community and cope with the stresses and demands that are found there. It is important that the child not become "institutionalized"-able to cope with the life style of hospital routines but unable to meet the challenges provided by the larger, more complex environment outside. Community excursions often provide the best test of a patient's progress in treatment. Further, they are very often crucial (especially in the later stages of treatment) in providing the patient with the corrective experiences he will need for successful reintegration into his own community upon discharge. Examples of these activities include movies, public swimming, parks, picnics, Y classes, public school classrooms, 
shopping trips, zoos, and museums. As should any parent, so must the primary staff encourage the child to become autonomous and independent, leaving the hospital nest at times to test his wings.

In order to minister to the developmental needs of a child, who may remain hospitalized for a year or more, consideration and attention must be given to setting and attaining goals for him. The staff must be knowledgeable about his patient's capabilities as well as his limitations as they relate to both the patient's pathology and his areas of health. His progress in treatment will always be, to some extent, uneven (functioning at age level in some areas, above or below in others), variable (i.e., unexpected sprints in ego integration, fixated plateaus, and periods of temporary regression), and unpredictable (due to sudden change in family situation, unexpected traumatic or fortuitous events, etc.). Therefore, it is important that goals be made flexible and that each contain a short- and long-term component. This, of course, implies that treatment strategies also be flexible and variable, even though sometimes they might seem at first glance contradictory or inconsistent.

A major role of the surrogate parent, as might be expected on an inpatient psychiatric unit, is that of behavior management. In a unit where the children are permitted certain freedom to "act out" their emotional conflicts, there are frequent and repeated demands placed upon the P.C. to monitor and intervene into the patient's behavior when such behavior is inappropriate, maladaptive, dangerous to himself or others, disruptive to the milieu, or otherwise problematic and/ or disturbed. Depending upon the specific pathology of the patient as well as numerous environmental factors, this may entail a major portion of the staff's work with the child, especially at the beginning.

A final role that parents perform in the rearing of their children and that the surrogate parent must also perform is that of representing the child's interest in issues involving interaction between the child and people outside the family (e.g., adults and children in the neighborhood school, YMCA, etc.). In the hospital, this need is readily apparent in the course of the day-to-day matters of administrative routines, as well as in the inevitable conflicts and disputes that arise between the staff or his child and other members of the milieu. It may involve nothing more than deciding whose toy is whose or who shall be held responsible for cleaning up a messy shower room; or it may involve more vital issues, such as how to deal with an incident of running away from the hospital or stealing a staff's wallet. In all such cases, the staff must determine his own child's best interests fairly and advocate decisions that will benefit the patient as well as respect 
the integrity and rights of others in the milieu. As primary staff, he must be the child's primary source of emotional support in the therapeutic milieu.

\section{Other Functions of the Primary Caretaker}

All of the other functions of the primary caretaker have been referred to as surrogate parent roles, i.e., functions performed in the absence of the child's natural parents in order to maintain adequate parenting. There are under the primary caretaker system at C.P.H. additional functions performed by the patient's staff on his behalf. These facilitate wise decision making within the team, maintain adequate documentation on the child's progress and/or lack thereof, and ensure that the child's wishes and feelings are communicated and respected.

The first two functions involve representation of the child's interest at team meetings and Developmental Profile meetings, where major policy decisions are reached with regard to many aspects of case management and treatment of the patient. As a liaison between the team and child, it is the primary caretaker's job to articulate the patient's needs and wishes at these meetings and in turn to communicate the corresponding decisions of the team back to the child. Equally important liaison work must be done by the primary staff between the patient and his parents and between the patient's parents and the team.

The primary staff is responsible for maintaining written documentation in several forms during his patient's treatment. These include preadmission information, daily behavior charting, monthly progress reports, a milieu care plan, and Developmental Profile ward reports. Before the patient is admitted to the hospital, a home visit is made by the assistant head nurse and the person who has been assigned to be the primary staff for the case. The purpose of the home visit is to establish an initial rapport with the patient and his family in the setting most familiar and comfortable to them, to inform the patient and family in general what living at C.P.H. will be like, to answer any questions they may have regarding the institution, to help minimize the stress and anxiety that members of the family may be experiencing around the admission, and to collect baseline data from the family covering a wide variety of areas. This includes the patient's medical history, personal hygiene habits, special interests, and hobbies. Also documented are the staff's initial perceptions regarding socioeconomic background, patterns of family interaction (e.g., patterns of 
parental limit setting and behavior management and the patient's response to these), and any other impressions that emerge from the visit that seem relevant. The information obtained from this preadmission home visit is then used to write a "Data Base" report, which becomes a permanent part of the patient's C.P.H. file.

Upon admission, daily charting is begun and maintained for each patient for the duration of his hospitalization, in compliance with federal and state law and in conformance with the standards set by the hospital itself. Charting at University Hospital by the nursing staff follows a procedure established by nursing personnel to meet these standards and involves a problem-oriented approach utilizing the "SOAP" format (i.e., subjective and objective analysis and plan). Accordingly, each patient's day is documented with regard to specifically defined behavioral problems. These are described in a problem list prepared by the patient's primary staff in consultation with the other members of the team. At the end of each evening's shift, the primary staff assesses the child's day, then describes his behavior and how the child seemed to experience it himself. The staff then analyzes the behavior and describes the plan used to manage it. If a new problem arises during the course of treatment, it is added to the problem list. When a problem on the list is considered resolved, this too must be documented. Daily charting records are legal documents and therefore are another part of the patient's permanent file.

Monthly case progress summary reports are written for every patient and submitted to the head nurse, who then uses them as reference material in her consultations with the inpatient director. They contain the primary staff's assessment of overall case progress, emphasizing the degree of effectiveness with which the case is being managed by the various members of the team with regard to their specific roles. Assessment is also made as to the overall functioning efficiency of the team with regard to cooperation, coordination of programs, communication of relevant data, and the like. These reports are not part of the patient's permanent record.

Within the first 6 weeks following a new patient's admission to the hospital, his primary'staff prepares a "Ward Care Plan." In addition to the child's vital statistics, a personality assessment describing strengths, weaknesses, needs, and behavioral problems is included in the plan. The plan also contains an outline of the interventions and management techniques being employed to provide the child with the behavioral limits, stimulation for development, external controls, and other elements of good parenting appropriate for him. Additional sections are included containing short- and long-term milieu treat- 
ment goals and any other information pertinent to parenting the child while he is living at C.P.H. The Ward Care Plan serves as a document of the individualized milieu therapy strategies specifically designed by the primary staff for each of his children. It is destroyed when the patient is discharged.

Finally, the primary staff must submit biannual ward reports to the child's psychotherapist for incorporation into the previously described Developmental Profiles. Ward reports contain information about the child's overall progress and response to the milieu therapy he has received on the ward since the previous Developmental Profile. They cover such areas as frustration tolerance, ability to defer gratification, response to limit setting, self-esteem, relationships with adults and peers, phase dominant activities, and others. Since the Profile is psychoanalytic in form and language, the supplemental report should also be conceptualized and written in a form that is compatible with and complementary to this format.

\section{Conclusion}

As can be seen, the inpatient service at the University of Michigan's Children's Psychiatric Hospital is a multidisciplined program whose purpose is to create a therapeutic milieu for its patients in which their emotional disturbances can be treated on many levels while many developmental variables are controlled. With this approach, it is hoped that the patient's stay at C.P.H. will permit the therapeutic management of all facets of his disturbance and result in the most efficient treatment possible. Implicit in these goals are the ideals of the speediest possible resolution of the child's emotional problems and the successful reintegration of the child into his family and the community.

It should also be clear that the work of the primary staff as milieu therapist is a key factor in the success or failure of any case in which he or she is involved. The primary staff's responsibilities are many and his influence extensive. He is a surrogate parent, a team member, a liaison between child and team and between child and parent. In addition, while the child is at C.P.H., the primary staff is the chief documentor of much of the child's behavior on the ward and in the community. A primary staff has responsibility for collecting behavioral data and communicating (verbally and in writing) to other members of the team problems encountered in managing the child's behavior, as well as progress made in these areas by various management and intervention techniques that he learns (or discovers) and then implements. 
In order to efficiently perform the many tasks expected of him as primary staff, it is clear that the P.C.W. (milieu therapist) should have expertise in many areas. These include exceptional observation, communication, and writing skills; knowledge of scientific methodology and psychoanalytic, child development, and learning theory; and, above all, exceptional parenting skills. Due to certain factors alluded to earlier (economics, present job requirements, administrative structure, theoretical orientation, etc.), however, such expertise is not a consistent norm. Moreover, to the extent to which such expertise is not found to be the norm, patient care suffers.

As a P.C.W., I intend to address myself further to some of the issues raised here, in an effort to examine (by case study) the results obtainable from efficient milieu therapy. In addition, I would like to look at the present barriers to attaining the ideal of the professional milieu therapist, and I hope to offer some viable solutions to the problems currently encountered in trying to reach this ideal.

\section{References}

1. Freud A: The Writings of Anna Freud. New York, International Universities Press, 1965.

2. Freud A, Nagera H, Bolland J: Anna Freud's Developmental Profile modifications and present form. Unpublished paper; part of the study entitled Assessment of Pathology in Childhood, conducted at the Hampstead ChildTherapy Clinic, London.

3. Nagera $\mathrm{H}$ : The primary caretaker system: A method for running in-patient units for children. Unpublished paper.

4. Lavatelli C, Stendler F: Readings in Child Behavior and Development. New York, Harcourt Brace Jovanovich, 1972.

5. Piaget J, Inhelder B: The Psychology of the Child. New York, Basic Books, 1969. 